\title{
Non-syndromic cleft palate: analysis of TBX22 exon 5 gene mutation
}

\author{
Run-Song Jiang, Xiong Zhao, Rui Liu
}

Children's Hostipal of Zhejiang University School of Medicine, Hangzhou, China

Submitted: 12 October 2010

Accepted: 21 April 2011

Arch Med Sci 2012; 8, 3: 406-410

DOI: $10.5114 /$ aoms.2012.28812

Copyright $\odot 2012$ Termedia \& Banach

\section{Abstract}

Introduction: This study aimed to investigate the mutation of T-box transcription factor TBX22 exon 5 in children with non-syndromic cleft palate. Four mutations in TBX22 exon 5 in X-linked cleft palate with ankyloglossia (CPX) patients had been identified in the previous studies. The study used the syndromic cleft palate susceptibility gene as a candidate gene for more common non-syndromic cleft palate.

Material and methods: A family-based study with parents and their children composing parent-child trios was performed in this research. Twenty children with non-syndromic cleft palate and 38 healthy parents were enrolled. TBX22 exon 5 was examined by polymerase chain reaction-single strand conformation polymorphism (PCR-SSCP) and DNA sequencing. The peaks of the sequence diagrams were analyzed using chromas 221 and the results of sequencing were proofread using dnastar6.13. The index of the transmission disequilibrium test (TDT) was calculated through McNemar testing.

Results: We have not found the presence of any mutation of TBX22 exon 5 reported in syndromic cleft palate patients in references. The index of TDT was 0.56 and showed no statistically significant difference $(p<0.05)$. No TBX22 exon 5 mutation was found in the 20 children.

Conclusions: Mutation of TBX22 exon 5 is not associated with non-syndromic cleft palate in the population of Jiangzhe areas in China.

Key words: non-syndromic cleft palate, TBX22, transmission disequilibrium test.

\section{Introduction}

Cleft lip and/or cleft palate is a common birth defect with a reported prevalence of 1 in 700 births worldwide [1]. The aetiology is complex and is thought to involve both major and minor genetic influences with variable interactions from environmental factors. The embryology of cleft lip and of cleft palate are largely distinct. Where one or more additional features are involved, clefts are referred to as syndromic; according to any associated anomalies, cleft palate is divided into syndromic and non-syndromic cleft palate. Significant progress has been made with the identification of gene mutations by studying syndromic cleft palate lately [2]. Some important findings have recently come from studies involving syndromic forms of the disorder. These include several genes which have now been shown to contribute a major effect to the aetiology. These genes can also be used to demonstrate a significant overlap between syndromic and non-syndromic clefts. It is clear that mutations in these genes may con-

\author{
Corresponding author: \\ Run-Song Jiang MD \\ Children's Hospital \\ Zhejiang University \\ School of Medicine \\ No. 57 Zhugan Lane \\ 310003 Hangzhou \\ Zhejiang, China \\ Phone: 8615924169177 \\ E-mail: jiangrs97@gmail.com
}


tribute to a proportion of the non-syndromic cleft cases [3].

X-linked cleft palate with ankyloglossia (CPX) (MIM 303400) is syndromic and inherited as a Mendelian, semi-dominant $\mathrm{X}$-linked disorder affecting male patients and approximately one third of female carriers having been described in several large families from different ethnic origins. Recently the underlying genetic defect in CPX was identified, where unique mutations were found in the T-box-containing transcription factor TBX22. TBX22 mutations underline a chromosome $X$-linked syndrome with cleft palate and ankyloglossia. The cleft phenotype predominantly affects males, who show variation ranging from a complete cleft of the secondary palate, submucous cleft, or bifid uvula to high arched palate. Although ankyloglossia is a common phenotype associated with TBX22 mutations, its occurrence is not a prerequisite for cleft palate and the two features are independent effects of the loss of TBX22 function. Mutations within families could result in either cleft palate only, ankyloglossia only, or both. The T-box transcription factor TBX22 is essential for normal craniofacial development, as demonstrated by the finding of nonsense, frameshift, splice-site, or missense mutations in patients with CPX. TBX22 mutations are reported in well-defined CPX families and in 4-8\% of non-syndromic cleft palate patients. Four mutations in TBX22 exon 5 in CPX patients were identified including three missenses and one small deletion. It has been recently demonstrated that TBX22 makes a significant contribution to prevalence of cleft palate at least in the Brazilian, North American and Thai cohorts. [4-7] The objectives of the study were to investigate whether TBX22 exon 5 mutation might be responsible for the formation of non-syndromic cleft palate in Chinese.

\section{Material and methods}

\section{Patients}

This was a family-based study with parents and their children composing parent-child trios. Informed consent was obtained from the parents before the study and it was approved by the Ethical Committee of Zhejiang University. We randomly selected 20 cases from 50 patients with non-syndromic cleft palate who were treated in our hospital, excluding single-parent families. A total of 20 children with non-syndromic cleft palate, with the exception of one who had repaired cleft lip, and 38 parents participated in this study. There was a twin in the team. The patients were aged from 12 to 36 months (average: $18.2 \pm 6.2$ months): 9 were boys and 11 girls. The cleft palate patients were divided into a bifid uvula or soft cleft palate group ( 8 persons) and a soft and hard cleft palate group (12 persons). Their mothers were aged from 22 to 36 years (average: $28.1 \pm 4.3$ years) and fathers aged from 25 to 40 years (average: $30.1 \pm 4.6$ years); they all denied a positive family history except one.

For each patient, a detailed phenotype was obtained. Cleft palate patients with ankyloglossia syndrome, Pierre-Robin syndrome, Stickler syndrome, chromosomal anomalies and kabuki make-up syndrome were excluded from the study.

\section{Amplification and sequencing}

Fifty-eight blood samples $(1 \mathrm{ml})$ were obtained. DNA was extracted from blood samples according to standard procedures. Primers were designed for genomic amplification and sequencing of exon 5 as described previously [5]. The primers were as follows: forward: 5'-ACATGGTGGAGGTGGTCAGGA-3'; reverse: 5'-ACATATCCCCTTGTGTAGTA-3'. The PCR reaction system (total $30 \mu \mathrm{l}$ ) contained $2.0 \mu \mathrm{l}$ of genomic DNA, $3.0 \mu \mathrm{l}$ of each primer, $1.0 \mu \mathrm{l}$ of each dNTP, $3.0 \mu \mathrm{l}$ of $\mathrm{Mg}^{2+}, 2 \mathrm{U}$ of Taq DNA polymerase, $3.0 \mu \mathrm{l}$ of $10 \times$ buffer.

Each PCR product $(10 \mu \mathrm{l})$ was subjected to conventional non-denaturing polyacrylamide gel electrophoresis and silver staining before observation and recording the results. The PCR products were purified with Millipore membrane and $3 \mu$ l of purified product was sequenced using a MegaBACE 1000 sequencing instrument.

\section{Sequence comparison and analysis}

The data of 58 cases of PCR product were measured, and the peaks of the sequence diagrams were analysed using chromas221 (version 2.21 technelysium). The results of sequencing were proofread using dnastar6.13. Statistical tests were based on two-sided probabilities with a significant $\alpha<0.05$. Baseline differences between parent-child trios were using the transmission disequilibrium test (TDT). Finally, the index of the transmission disequilibrium test (TDT) was calculated through McNemar testing. The TDT is a useful method to detect linkage between a marker locus and a disease susceptible locus. It is not prone to producing false positive results due to population stratification [8].

\section{Results}

In the family-based study of 58 samples, we have detected the sequences of the child team $(n=20)$ and the parents team $(n=38)$. Especially, we have checked the sequences of the family with a twin. Sequencing results were aligned with the standard sequence of TBX22 exon 5 in GenBank. We have found no mutations in TBX22 exon 5 in our research, though four mutations were identified in TBX22 exon 5 in CPX patients in previous studies. The first one's codon change was 


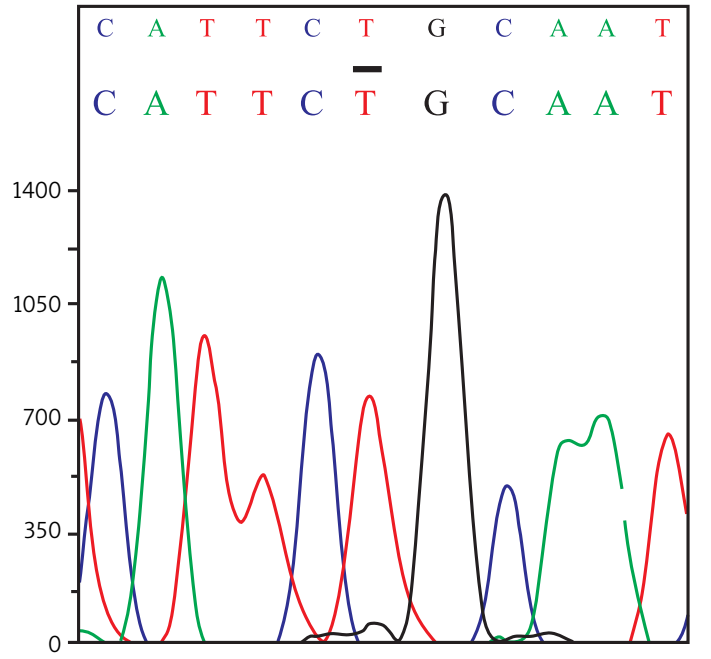

Figure 1. Non-syndrome cleft palate children and the mutation of T-box transcription factor TBX22 exon 5: there is no missense mutation $(\mathrm{T} \rightarrow \mathrm{C})$

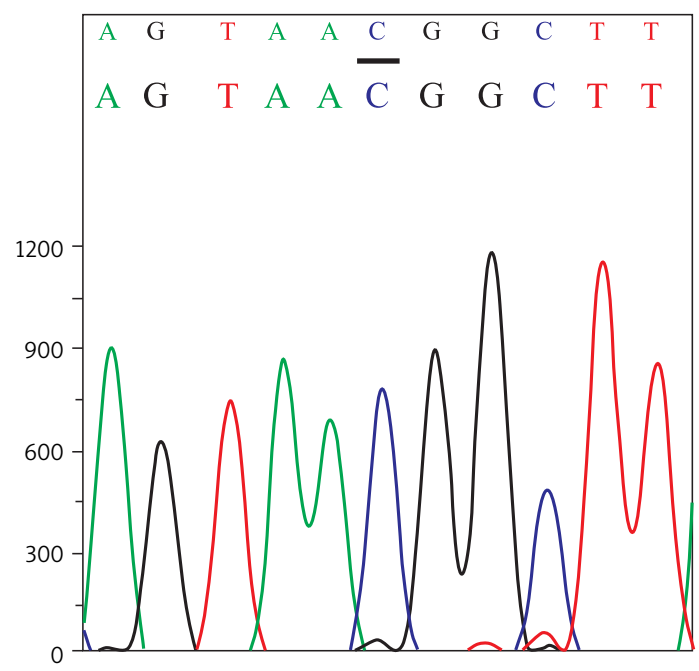

Figure 3. Non-syndrome cleft palate children and the mutation of T-box transcription factor TBX22 exon 5: there is no missense mutation $(C \rightarrow T)$

$641 \mathrm{~T} \rightarrow \mathrm{C}(\mathrm{CTG} \rightarrow \mathrm{CCG})$, amino acid change was Leu $\rightarrow$ Pro(L214P) and its accession number was CM022464 [4]; the second one's codon change was 671delC (AGTAC^AAACCCCGAGTGCACG) and its accession number was CD013205 [5]; the third one's codon change was $785 \mathrm{C} \rightarrow \mathrm{T}(\mathrm{ACG} \rightarrow \mathrm{ATG})$, amino acid change was $\mathrm{Thr} \rightarrow$ Met (T260M) and its accession number was CM013049 [5]; the last one's codon change was 790A $\rightarrow$ T (aAAC $\rightarrow$ TAC), amino acid change was Asn $\rightarrow$ Tyr (A264T) and its accession number was CM040122 [6]. In the corresponding base site of TBX22 exon 5, we found 646T, 671C, 785C and 796A, which can be seen in the pictures below (Figures 1-4). There were no missense mutations or small deletions identified. No clinical sig-

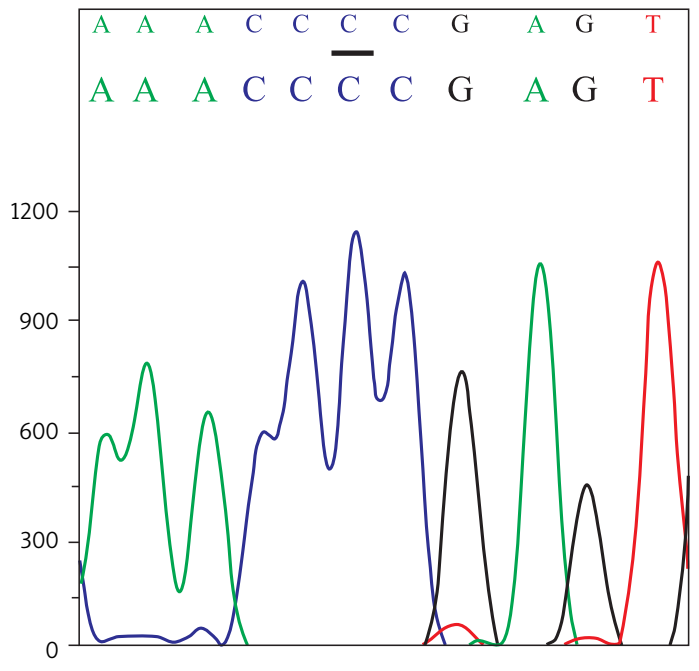

Figure 2. Non-syndrome cleft palate children and the mutation of T-box transcription factor TBX22 exon 5: there is no small deletion (delC)

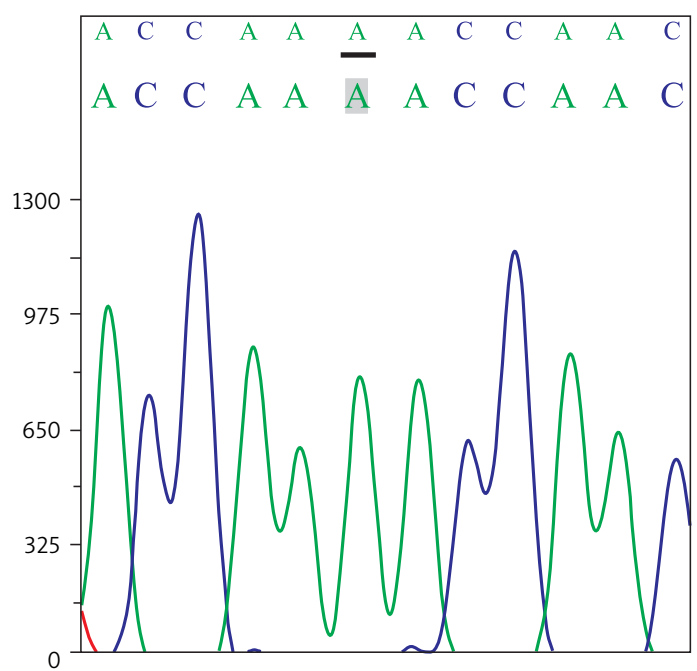

Figure 4. Non-syndrome cleft palate children and the mutation of T-box transcription factor TBX22 exon 5 : there is no missense mutation $(A \rightarrow T)$

nificance was found in calculating the index of the transmission disequilibrium test of 20 parent-child trios $(p>0.05)$ and the index of TDT was 0.56 .

\section{Discussion}

In our study we used the syndromic cleft palate susceptibility gene as a candidate gene for more common non-syndromic cleft palate. Efforts to identify the genetic factors have been most successful for monogenic, syndromic clefts, such as in Van der Woude syndrome (involving IRF6); Margarita Island syndrome (PVRL1); Kallmann syndrome (FGFR1); ectrodactyly, ectodermal dysplasia, and cleft lip and/or palate syndrome (p63); cleft lip and/or 
palate with hypodontia (MSX1); and X-linked cleft palate (TBX22) [3, 9]. The value of translating a mutation causing a rarely observed syndromic condition onto more common non-syndromic forms of the disorder has been elegantly demonstrated in the study by Sözen et al. [10] for the role of PVRL1 in cleft lip with or without cleft palate. Phenotypic penetrance within families is often variable, and has not been explained by the type or position of mutations within the genes [11].

Considerable success has been achieved in identifying aetiological genes for syndromic forms of orofacial clefting with approximately $15-20 \%$ cloned to date. TBX22 is the important susceptibility gene currently found in X-linked cleft palate - ankyloglossia syndrome (CPX) and it is a major genetic determinant for familial cleft palate, particularly when combined with ankyloglossia. Linkage and/or haplotype analysis has been used to demonstrate that the cleft palate and ankyloglossia phenotype was $X$-linked and mapped to the approximate interval of CPX in Xq21 [4, 5, 12-14]. As a transcription factor, TBX22 is expressed specifically in tissues related to palatogenesis and it is confirmed as a major genetic influence in normal palate development. TBX22 expression was reported to disappear just prior to palatal shelf fusion and was thought instead to be required for mesenchymal proliferation and shelf elevation. Identification of the downstream target genes remains a priority $[3,6,15]$.

TBX22 clearly makes a significant contribution to the prevalence of cleft palate and it will be important to study its biological role in the process of craniofacial development. To date, 13 different TBX22 mutations have been reported in CPX patients (http://www.hgmd.cf.ac.uk), including missense, nonsense, splicing, regulatory, small deletions and small insertions. There were four mutations located in TBX22 exon 5: 641T $\rightarrow$ C(L214P) [4], 671delC(221) [5], 785C $\rightarrow$ T(T260M) [5], 790A $\rightarrow$ T(A264T) [6]. In our research the sequence alignment did not reveal any credible mutation. We have not examined all the exons of TBX22, so mutations may also be harboured in the other exons. Suphapeetiporn et al. [7] in their study performed mutation analysis that covered all the coding regions of the TBX22 gene in 53 unrelated Thai patients with non-syndromic cleft palate, and identified four potentially pathogenic mutations: $359 \mathrm{G} \rightarrow \mathrm{A}(\mathrm{R} 120 \mathrm{Q}), 452 \mathrm{G} \rightarrow \mathrm{T}$ $(\mathrm{R} 151 \mathrm{~L}), 1166 \mathrm{C} \rightarrow \mathrm{A}(\mathrm{P} 389 \mathrm{Q})$ and 1252 delG in four different patients. There were also no mutations in exon 5 in the Thai population with non-syndromic cleft palate.

A better understanding of the cellular biology will help us to afford appropriate advice, therapy and ultimately prevention. A missense variant (359G $\rightarrow$ A; R120Q) was detected in the Thai population with ankyloglossia alone, the same mutation being previously reported in Thai patients with non-syndromic cleft palate. This nucleotide substitution was next to the one that has been described in a Tunisian CPX family (358C $\rightarrow$ T; R120W). From the crystal structure of the Brachyury $T$ domain, this position lies within the $\alpha \beta$ barrel and is predicted to be involved in DNA binding. The amino acid of another missense variant in a CPX family $(790 \mathrm{~A} \rightarrow \mathrm{T}$; A264T) is located in the T box domain at the start of the $\alpha$ helix 3 , which is the position to recognise the target DNA in the minor groove and bridges the DNA backbone. Interfering with DNA binding is likely to be one of the major mechanisms underlying cleft palate with or without ankyloglossia as evidenced by a number of missense mutations found in the DNA-binding domain of TBX22 [6, 7, 14-16].

Based on the family-based study which refers to the patients and their healthy parents, the parent-child trio becomes the research object. Transmission of a particular allele from heterozygous parents to an offspring is expected to be approximately $50 \%$, when there is no association of the allele and the disease. If the allele is associated with an increased risk of the disease, excess transmission to the offspring is expected. This approach accounts for the non-independence of parental transmissions to affected offspring $[17,18]$. The suspected correlation was subjected to validation using the transmission disequilibrium test. The allele is not transmitted to affected children. As a control with a pair of alleles for a 1:1 matched case-control study, TDT eliminates the case-control population stratification problem, largely avoiding the phenomenon of the occurrence of false positives and improving the accuracy of gene targeting [19-22].

In conclusion, we found no mutations in TBX22 exon 5 in our patients with non-syndromic cleft palate, but the sample size is limited, and mainly concentrated in the selection of patients within Jiangzhe areas in China. As coverage is not wide enough, these restrictions may have some influences and induce negative results.

\section{Acknowledgments}

We thank all patients and their parents for participation. This work was performed in cooperation with HuaDa gene company of Zhejiang University and supported by the health bureau of Zhejiang province. We also thank Debra Marouth for critical reading of the manuscript.

Contributors: Run-song Jiang and Xiong Zhao collected, analyzed and interpreted the data. Run-song Jiang drafted the manuscript for the final version to be published. Rui Liu, a research guide, designed and did the critical revision of the manuscript.

\footnotetext{
References

1. Bender PL. Genetics of cleft lip and palate. J Pediat Nurs 2000; 15: 242-9.
} 
2. Carinci F, Scapoli L, Palmieri A, Zollino I, Pezzetti F. Human genetic factors in nonsyndromic cleft lip and palate: an update. Int J Pediatr Otorhinolaryngol 2007; 71: 1509-19.

3. Stanier P, Moore GE. Genetics of cleft lip and palate: syndromic genes contribute to the incidence of nonsyndromic clefts. Hum Mol Genet 2004; 13: R73-81.

4. Braybrook C, Lisgo S, Doudney K, et al. Craniofacial expression of human and murine TBX22 correlates with the cleft palate and ankyloglossia phenotype observed in CPX patients. Hum Mol Genet 2002; 11: 2793-804.

5. Braybrook C, Doudney K, Marçano AC, et al. The T-box transcription factor gene TBX22 is mutated in X-linked cleft palate and ankyloglossia. Nat Genet 2001; 29: 179-83.

6. Marçano AC, Doudney K, Braybrook C, et al. TBX22 mutations are a frequent cause of cleft palate. J Med Genet 2004; 41: 68-74.

7. Suphapeetiporn K, Tongkobpetch S, Siriwan P, Shotelersuk V. TBX22 mutations are a frequent cause of nonsyndromic cleft palate in the Thai population. Clin Genet 2007; 72: 478-83.

8. Purcell S, Cherny SS, Sham PC. Genetic Power Calculator: design of linkage and association genetic mapping studies of complex traits. Bioinformatics 2003; 19: 149-50.

9. Murray JC, Schutte BC. Cleft palate: players, pathways, and pursuits. J Clin Invest 2004; 113: 1676-8.

10. Sözen MA, Suzuki K, Tolarova MM, Bustos T, Fernández Iglesias JE, Spritz RA. Mutation of PVRL1 is associated with sporadic, non-syndromic cleft lip/palate in northern Venezuela. Nat Genet 2001; 29: 141-2.

11. Pauws E, Moore GE, Stanier P. A functional haplotype variant in the TBX22 promoter is associated with cleft palate and ankyloglossia. J Med Genet 2009; 46: 555-61.

12. Carlson CS, Eberle MA, Kruglyak L, Nickerson DA. Mapping complex disease loci in whole-genome association studies. Nature 2004; 429: 446-52.

13. Andreou AM, Pauws E, Jones MC, et al. TBX22 missense mutations found in patients with $\mathrm{X}$-linked cleft palate affect DNA binding, sumoylation, and transcriptional repression. Am J Hum Genet 2007; 81: 700-12.

14. Chaabouni M, Smaoui N, Benneji N, et al. Mutation analysis of TBX22 reveals new mutation in Tunisian CPX family. Clin Dysmorphol 2005; 14:23-5.

15. Kantaputra PN, Paramee M, Kaewkhampa A, et al. Cleft lip with cleft palate, ankyloglossia, and hypodontia are associated with TBX22 mutations. J Dent Res 2011; 90: 450-5.

16. Müller CW, Herrmann BG. Crystallographic structure of the T domain-DNA complex of the Brachyury transcription factor. Nature 1997; 389: 884-8.

17. Ferreira MA, Sham P, Daly MJ, Purcell S. Ascertainment through family history of disease often decreases the power of family-based association studies. Behav Genet 2007; 37: 631-6.

18. Niemiec P, Zak I, Emich-Widera E, et al. The C242T polymorphism of the gene encoding cytochrome b-245 alpha is not associated with paediatric ischaemic stroke: familybased and case-control study. Neurol Neurochir Pol 2010; 44: 453-8.

19. Eaves LJ, Sullivan P. Genotype-environment interaction in transmission disequilibrium tests. Adv Genet 2001; 42: 223-40.

20. Ichikawa E, Watanabe A, Nakano Y, et al. PAX9 and TGFB3 are linked to susceptibility to nonsyndromic cleft lip with or without cleft palate in the Japanese: population-based and family-based candidate gene analyses. J Hum Genet 2006; 51: 38-46.

21. Mitchell LE, Beaty TH, Lidral AC, et al. Guidelines for the design and analysis of studies on nonsyndromic cleft lip and cleft palate in humans: summary report from a Workshop of the International Consortium for Oral Clefts Genetics. Cleft Palate Craniofacial J 2002; 39: 93-100.

22. Xu YL, Sun Q, Shan GL, et al. A case-control study on risk factors of breast cancer in China. Arch Med Sci 2012; 8: 303-9. 